\title{
Véronique Donard, Du meurtre au sacrifice, psychanalyse et dynamique spirituelle
}

Paris, Éditions du Cerf, 2009, 671 p.

\section{Benoît Vermander}

\section{OpenEdition}

Journals

Édition électronique

URL : http://journals.openedition.org/assr/22892

DOI : $10.4000 /$ assr.22892

ISSN : $1777-5825$

Éditeur

Éditions de l'EHESS

Édition imprimée

Date de publication : 31 décembre 2011

Pagination : 158

ISBN : 9782713223273

ISSN : 0335-5985

Référence électronique

Benoît Vermander, « Véronique Donard, Du meurtre au sacrifice, psychanalyse et dynamique spirituelle », Archives de sciences sociales des religions [En ligne], 156 | octobre-décembre 2011, document 156-43, mis en ligne le 15 février 2012, consulté le 21 septembre 2020. URL : http:// journals.openedition.org/assr/22892 ; DOI : https://doi.org/10.4000/assr.22892

Ce document a été généré automatiquement le 21 septembre 2020.

(c) Archives de sciences sociales des religions 


\title{
Véronique Donard, Du meurtre au sacrifice, psychanalyse et dynamique spirituelle
}

\author{
Paris, Éditions du Cerf, 2009, 671 p.
}

\section{Benoît Vermander}

\section{RÉFÉRENCE}

Véronique DoNARD, Du meurtre au sacrifice, psychanalyse et dynamique spirituelle, Paris, Éditions du Cerf, 2009, 671 p.

1 Ambitieux essai de métapsychologie, cet ouvrage entend rendre compte de la dimension spirituelle de l'être humain selon une approche psychanalytique rigoureusement ancrée dans la tradition freudienne; il veut aussi montrer que cette dimension collabore activement à la structuration et au développement psychiques; il fait de la notion de "sacrifice » le fil conducteur du développement psychique dont il est ici question, postulant, aux côtés du sadisme et du masochisme originaires, une troisième position du moi, "l'organisation sacrificielle originaire», laquelle (en fonction du mode de gestion par le sujet du traumatisme primordial de la désaide du nourrisson comme de ses éventuelles défaillances environnementales) peut s'avérer pathologique (le cas extrême étant celui du «meurtre sacrificiel » en série) ou bien donner naissance à une logique spirituelle faisant $\mathrm{du}$ « sacrifice de soi » une dynamique de vie plutôt qu'un acte de mort. Enfin, par le fait même d'insérer entre les deux parties consacrées, d'une part à la pathologie, de l'autre à l'assomption de la position sacrificielle, trois chapitres centrés sur l'analyse de la logique sacrificielle dans différentes traditions religieuses, l'auteure se risque à une approche conjointe de l'anthropologie du fait religieux et de la métapsychologie, approche menée en fonction de concepts opératoires unifiés. Elle va jusqu'à suggérer de rechercher l'origine 
biologique du modèle proposé dans le « suicide cellulaire altruiste » (apoptose) théorisé notamment par Jean-Claude Ameisen.

L'ampleur du projet ne peut que susciter tout à la fois un vif intérêt et de nombreuses questions. Les dimensions de l'ouvrage et sa structure très méthodique permettent à l'auteure de se mesurer avec bon nombre d'entre elles, d'une façon qui souvent dissipe doutes et malentendus, mais qui parfois aussi les aiguise. De façon générale, la maitrise montrée par V. Donard dans la lecture de l'œuvre de Freud, de Mélanie Klein et de Winnicott lui inspire des interprétations tout à la fois rigoureuses et inventives des apports de ces derniers. Les psychanalystes contemporains sont également abondamment cités (larges emprunts à l'œuvre de Sophie de Mijolla-Mellor). Les chapitres consacrés à l'histoire et l'anthropologie du fait religieux sont beaucoup moins convaincants, et les études de cas sont trop souvent fondées sur de longues notes de lecture d'un ou deux ouvrages faisant autorité en la matière. Les riches analyses du texte biblique soulèvent d'autres questions, sur lesquelles je reviendrai plus avant.

Si les développements historiques et anthropologiques soulèvent des réserves, il n'en va pas de même des deux «cas personnels » qui ouvrent et ferment respectivement l'ouvrage. L'analyse d'un meurtre "sacrificiel " pour lequel l'auteure a été appelée à intervenir comme expert l'engage et nous engage fortement dans le sujet traité, et elle prépare l'analyse des traumatismes et pathologies psychiques qui forme bonne part de la première partie de l'ouvrage. Avec deux réserves pourtant: V. Donard s'interdisant pour des raisons éthiques de dévoiler nombre de détails concernant le premier cas analysé, ses inférences exigent parfois du lecteur quelque chose qui s'apparente à un «acte de foi». Par ailleurs, aussi stimulante que soit l'analyse des pathologies sacrificielles ensuite exposées, la question du caractère "universel " des processus ainsi décrits reste entière à ce stade. Ces réserves ne se retrouvent pas à la lecture de la longue analyse du «cas» de Thérèse de Lisieux qui clôt l'ouvrage: sur un sujet pourtant déjà abondamment traité, les pages consacrées à la jeune carmélite vont aussi loin qu'il est possible tant dans le rendu des pathologies sociales, familiales et personnelles de Thérèse que dans celui de l'extraordinaire phénomène de sublimation par lequel Thérèse opère sa "percée » spirituelle au travers de l'épaisseur même de sa condition. Ces pages finales ont été préparées bien plus tôt dans l'ouvrage par une excellente analyse des usages contemporains du concept surchargé de "sublimation ", analyse qui englobe celle de l'acte d'écrire comme stratégie utilisée tout à la fois pour accomplir et éviter le meurtre sacrificiel que l'organisation psychique du sujet écrivant appellerait (p. 184-193).

4 En contraste, la partie centrale laisse quelque peu perplexe : elle entend montrer que les mythes de création sont généralement fondés sur le récit d'un sacrifice donnant sa structure au cosmos et son sens à l'existence humaine, laquelle alors répèterait toujours quelque peu cet acte originel, inscrit peut-être jusque dans l'organisation cellulaire du vivant. Le chapitre premier de cette partie, consacré aux termes d'origine et de mythe, est fondé pour bonne part sur une lecture de Mircea Eliade, et marqué par le caractère parfois ancien de ses références. Le chapitre qui suit - une lecture des mythes védiques, orphiques et aztèques - semble illustrer l'extraordinaire diversité des pratiques, justifications, évolutions et interprétations de l'acte sacrificiel bien davantage que son unité primordiale, aux rebours de ce que voudrait l'auteure. Surtout, nulle place n'est accordée à une «logique du social» qui jouirait de quelque autonomie: le collage effectué entre histoire personnelle et récit collectif ignore la 
façon dont les stratégies collectives de création et manipulation du «sens » sont liées aussi à des types d'organisation sociale et étatique très fortement différenciés. Le long chapitre consacré à l'univers biblique est de facture différente. V. Donard y reprend les lectures de quelques récits structurants, ceux du meurtre d'Abel et du sacrifice d'Isaac principalement, mais aussi les chants du Serviteur souffrant (Is 50-52). Empruntant à des exégèses antérieures souvent bien connues, ces pages illustrent avec bonheur une dimension du corpus biblique. Mais nous sommes loin de faire ici le tour de la question, et la prise en compte d'autres textes (bonne part des psaumes et des écrits prophétiques, l'Épitre aux Hébreux) complexifierait considérablement les données du problème, en introduisant ce que l'on peut appeler la tradition « antisacrificielle » qui lie aussi en un tout l'un et l'autre Testament. Nous sommes donc là simplement en présence d'une lecture psychanalytique de quelques extraits du texte biblique, dans un style difficile à rapporter aux essais de synthèse qui marquait l'approche des autres traditions religieuses évoquées. Ces questions méthodologiques, qui grèvent l'ensemble de la deuxième partie, affaiblissent du coup la portée théorique de la troisième, dans laquelle l'auteure entend passer «de l'universalité du mythe à un fonctionnement psychique individuel» (p.449) - même si, comme nous l'avons déjà noté, le cas qui fonde entièrement cette partie, celui de Thérèse, offre quelques-unes des plus belles pages de l'ouvrage. L'unité même du dessein des première et troisième parties rend plus malaisée encore à accepter l'élargissement du dessein de l'ouvrage auquel s'essaie la partie centrale.

$5 \quad$ L'essentiel de ce dessein est repris et explicité dans la conclusion de l'ouvrage : la thèse est celle de l'existence d'un fantasme primordial, récurrent et universel, celui d'assister à un meurtre de facture sacrificielle, un fantasme où il est question de sacrifier ou d'être sacrifié. Fantasme nourri lui-même par le trauma primordial aux sources de l'organisation psychique, lequel est directement lié à l'imminence et l'évidence de la mort (p.624). Pour V. Donard, ce fantasme primordial ne fait pas nombre avec les autres (scène primitive, castration et séduction), mais s'y ajoute et les complète. La formulation de ce fantasme (il faut sacrifier pour) introduit une instance tiers, cause finale du sacrifice, lieu vide qui n'est pas identifiable au surmoi mais à une instance de type déique. Toute dynamique spirituelle prend sa source dans cette situation traumatique primordiale. En finale, «il n'appartient pas à la psychanalyse de se prononcer sur la réalité ou non de ce qui, à la source du vécu spirituel ou religieux, peut être nommé "Dieu". » (p. 630)

6 Cette recension ne fait pas justice à la richesse de certaines pages de l'ouvrage, par exemple sur l'évolution continue de la théorie des pulsions chez Freud et ses successeurs. Mais ces analyses ne sont pas toutes de la même eau, et l'ouvrage souffre en plusieurs endroits de lourdeurs didactiques comme de la valeur un peu inégale de ses références. Quoi qu'il en soit, il prépare avec prudence et méthode l'énoncé d'une hypothèse de grande portée. Il convient toutefois de noter : a) que cette hypothèse ne prend sens qu'à l'intérieur de la théorie freudienne dont elle se réclame, et qu'elle perd toute cohérence interne dès qu'elle en est détachée; b) qu'elle tend à aplatir la diversité des vécus culturels, religieux et sociaux en "absolutisant» le contenu et la portée de la notion qu'elle analyse. Le courage qu'il est nécessaire de déployer pour se mesurer de front à pareil sujet comme le dispositif mis en place pour soutenir et nourrir au plus près l'argumentation n'en font pas moins de cet ouvrage un essai d'ampleur remarquable. 